Research Paper

\title{
The relative efficacy and safety of targeted agents used in combination with chemotherapy in treating patients with untreated advanced gastric cancer: a network meta-analysis
}

\author{
Shuping Xie ${ }^{1, *}$, Huixiang Zhang ${ }^{2, *}$, Xueyan Wang ${ }^{1}$, Quanxing $\mathbf{G e}^{1}$, Junhong $\mathbf{H u}^{3}$ \\ ${ }^{1}$ Department of Gastroenterology, Huaihe Hospital of Henan University, Kaifeng, Henan, 475000, China \\ ${ }^{2}$ Department of Radiotherapy, Huaihe Hospital of Henan University, Kaifeng, Henan, 475000, China \\ ${ }^{3}$ Department of General Surgery, Huaihe Hospital of Henan University, Kaifeng, Henan, 475000, China \\ *These authors contributed equally to this work
}

Correspondence to: Junhong Hu, email: tangyinhuatyh@126.com

Keywords: gastric cancer, targeted therapy, efficacy and safety, network meta-analysis

Received: November 10, 2016

Accepted: February 15, 2017

Published: March 06, 2017

Copyright: Xie et al. This is an open-access article distributed under the terms of the Creative Commons Attribution License (CC-BY), which permits unrestricted use, distribution, and reproduction in any medium, provided the original author and source are credited.

\section{ABSTRACT}

Gastric cancer is one of the leading mortal causes. Targeted therapy is a new type of cancer treatment, which precisely identifies and attacks cancer cells and significantly reduces side effects. In this network meta-analysis, we focused on the efficacy and safety of 12 targeted agents on gastric cancer among a total of 8,405 patients from 24 trials. Hazard ratio (HR) with 95\% credible interval (CrI) were calculated for primary outcomes, including overall survival (OS) and progression-free survival (PFS), while odds ratio (OR) with $95 \%$ CrI were calculated for secondary outcomes. Surface under the cumulative ranking curve (SUCRA) were calculated to illustrate the rank probability of various agents for different outcomes. Compared with other analyzed treatments, ramucirumab is outstanding in survival outcomes. However, higher risk of hematological events should be noted during its application. Lapatinib is also efficacious in progression reduction, while it is always combined with severe gastrointestinal events. Trastuzumab is proposed for its high efficacy in improving survival rate and safety, which is proper for most patients. In conclusion, trastuzumab was recommended as the optimal targeted agent combined with chemotherapy for gastric cancer patients.

\section{INTRODUCTION}

Gastric cancer is one of the leading causes of death, ranking the fourth in the prevalence of cancer. Globally, 723,100 deaths and 951,600 new cases occur each year [1]. Meanwhile, gastric cancer takes the third place in the global burden of disability-adjusted lifeyears, following lung and liver cancers [2]. Thanks to the improvement of dietary, sanitation and development of antibiotics, the incidence rate and mortality of gastric cancer has decreased recently. However, in developing countries, it remains to be a great threat to our life, with the 5-year survival rate less than $25 \%$ [3]. In addition to environment factors, gastric cancer with a specific gene and family background is also prevailing.

Chemotherapy has been proved to be an effective treatment for patients with advanced gastric cancer, and currently, combination of fluoropyrimidine with platinum or irinotecan, triplet combination of fluoropyrimidine/ platinum with docetaxel or epirubicin are provided as the standard first-line chemotherapy for patients with advanced gastric cancer [4-6]. However, it usually takes a few months to produce clinical therapeutic effect and patients often suffer from severe adverse effects including vomiting, nausea, rashes, headache, etc. Moreover, more than half of the patients may not response to the therapy [7]. To further improve the effect of chemotherapy and reduce corresponding toxicity, targeted therapy was introduced.

Targeted therapy is a new type of cancer treatment and a special type of chemotherapy. Agents used in targeted therapy can precisely identify and attack certain type of cancer cells based on the mutation of gene and protein. Meanwhile, little damage is done to normal cells, thus targeted therapy can significantly avoid side effects. Used in in combination with chemotherapy, targeted 
therapy is effective in many cancers. For example, Herceptin (trastuzumab), which targeted at breast cancers with the over-expression of Human Epidermal Growth Factor Receptor 2 (HER2), has been proved to significantly improve overall survival (OS) and disease-free survival (DFS) for both early-stage and advanced patients according to a 2014 Cochrane Review [8]. However, for patients with advanced gastric cancer, there is no standard targeted therapy, and this network meta-analysis was designed to evaluate the performance of different targeted drugs used in combination with chemotherapy and try to find out the most effective one/ones.

\section{RESULTS}

\section{Study characteristics}

A total of 8,405 patients from 23 RCTs were enrolled in our analysis and listed in Supplementary Table 1 [9-31]. Twelve regimens on 7 targets were involved, including ramucirumab, bevacizumab, nimotuzumab, cetuximab, lapatinib, trastuzumab, endostar, everolimus, matuzumab, onartuzumab, panitumumab and sunitinib. The process of selection was illustrated in flow chart as Figure 1.

Network plot in Figure 2 presented the comparisons of various treatments for different outcomes. The width of the lines is proportional to the number of trials comparing each pair of treatments and numbers on the lines illustrate the exact number; the area of circles represents the cumulative number of patients for each intervention.

\section{Overall survival}

The network meta-analysis results were listed in Supplementary Tables 2, 3 and Figures 3, 4. For 1y-OS, only trastuzumab and ramucirumab were found to be helpful compared to placebo (placebo vs. trastuzumab: $\mathrm{HR}=1.30$, 95\% CrI: 1.01-1.67; placebo vs. ramucirumab: $\mathrm{HR}=1.36$, 95\% CrI: 1.21-1.53). They were also superior than cetuximab, matuzumab and panitumumab (cetuximab vs. ramucirumab: $\mathrm{HR}=1.51,95 \% \mathrm{CrI}$ : $1.18-1.93$; cetuximab vs. trastuzumab: $\mathrm{HR}=1.44,95 \% \mathrm{CrI}$ : 1.03-2.01; matuzumab vs. ramucirumab: $\mathrm{HR}=2.13,95 \% \mathrm{CrI}: 1.45-3.12$; matuzumab vs. trastuzumab: $\mathrm{HR}=2.03,95 \% \mathrm{CrI}: 1.30$ 3.17; panitumumab vs. ramucirumab: $\mathrm{HR}=1.74,95 \% \mathrm{CrI}$ : 1.19-2.55; panitumumab vs. trastuzumab: $\mathrm{HR}=1.66,95 \%$ CrI: 1.07-2.59). Besides, matuzumab was less efficacious than most of treatments including placebo. As for $2 \mathrm{y}-\mathrm{OS}$, bevacizumab, lapatinib, ramucirumab and trastuzumab were observed to perform better than placebo (bevacizumab vs. placebo: $\mathrm{HR}=0.85,95 \% \mathrm{CrI}$ : 0.77-0.95; lapatinib vs. placebo: $\mathrm{HR}=0.84,95 \% \mathrm{CrI}$ : $0.74-0.95$; placebo vs. ramucirumab: $\mathrm{HR}=1.26,95 \% \mathrm{CrI}: 1.15-1.37$; placebo vs. trastuzumab: $\mathrm{HR}=1.32$, 95\% CrI: $1.15-1.52$ ). These four agents also performed better than cetuximab, nimotuzumab and panitumumab. Nimotuzumab showed worse efficacy than most treatments and placebo. Concerning 3y-OS, lapatinib was observed to have better perform than cetuximab (cetuximab vs. lapatinib: $\mathrm{HR}=1.21,95 \% \mathrm{CrI}$ : 1.04-1.41). Trastuzumab showed a significantly better efficacy than cetuximab, nimotuzumab and placebo (cetuximab vs. trastuzumab: HR $=1.44,95 \%$ CrI: $1.15-1.79$; nimotuzumab vs. trastuzumab: $\mathrm{HR}=1.68,95 \% \mathrm{CrI}: 1.10-2.57$; placebo vs. trastuzumab: $\mathrm{HR}=1.36,95 \% \mathrm{CrI}: 1.11-1.65)$.

\section{Progression-free survival}

Bevacizumab, everolimus, lapatinib, ramucirumab, trastuzumab and sunitinib were found to be efficacious in improving 1y-PFS compared to placebo (bevacizumab vs. placebo: $\mathrm{HR}=0.72,95 \% \mathrm{CrI}$ : 0.63-0.82; everolimus vs. placebo: $\mathrm{HR}=0.70,95 \% \mathrm{CrI}: 0.55-0.89 ;$ lapatinib vs. placebo: $\mathrm{HR}=0.78,95 \% \mathrm{CrI}$ : 0.64-0.94; placebo vs. ramucirumab: $\mathrm{HR}=1.73,95 \% \mathrm{CrI}: 1.52-1.98$; placebo vs. sunitinib: $\mathrm{HR}=1.32,95 \% \mathrm{CrI}: 1.02-1.69$; placebo vs. trastuzumab: $\mathrm{HR}=1.49,95 \% \mathrm{CrI}: 1.13-1.96)$. Ramucirumab was more efficacious than major portion of the treatments, which was consistent with the result in OS. And everolimus showed a good efficacy in 1y-PFS, better than cetuximab, matuzumab, nimotuzumab, onartuzumab, panitumumab as well as placebo (cetuximab vs. everolimus: $\mathrm{HR}=1.52,95 \% \mathrm{CrI}: 1.12-2.07$; everolimus vs. matuzumab: $\mathrm{HR}=0.46,95 \% \mathrm{CrI}: 0.31-0.67$; everolimus vs. nimotuzumab: $\mathrm{HR}=0.59,95 \% \mathrm{CrI}$ : 0.41-0.84; everolimus vs. ontaruzumab: $\mathrm{HR}=0.68,95 \%$ CrI: 0.47-0.98; everolimus vs. panitumumab: $\mathrm{HR}=0.68$, 95\% CrI: 0.47-0.98; everolimus vs. placebo: $\mathrm{HR}=0.70$, 95\% CrI: 0.55-0.89). However, matuzumab were not as efficacious as most treatments, meanwhile it was the only treatments performed worse than placebo. For $2 \mathrm{y}$-PFS, nimotuzumab performed worse than all the treatments except matuzumab and onartumumab. Endostar had a better effect than bevacizumab, cetuximab, lapatinib, matuzumab, nimotuzumab, onartuzumab and placebo (bevacizumab vs. endostar: $\mathrm{HR}=1.41,95 \% \mathrm{CrI}: 1.12-$ 1.76; cetuximab vs. endostar: $\mathrm{HR}=1.98,95 \% \mathrm{CrI}$ : 1.58-2.49; endostar vs. lapatinib: $\mathrm{HR}=0.70,95 \% \mathrm{CrI}$ : 0.55-0.88; endostar vs. matuzumab: $\mathrm{HR}=0.48,95 \%$ CrI: $0.26-0.88$; endostar vs. nimotuzumab: $0.25,95 \%$ CrI: 0.14-0.47; endostar vs. onartuzumab: $0.50,95 \%$ CrI: 0.31-0.79; endostar vs. placebo: $0.54,95 \% \mathrm{CrI}$ : 0.44-0.66). Moreover, ramucirumab were also superior to over half of the treatments. In 3y-PFS, both endostar and ramucirumab were significant helpful in progression reduced than other treatments. Cetuximab was less efficacious than any other treatments except placebo (bevacizumab vs. cetuximab: $\mathrm{HR}=0.60,95 \% \mathrm{CrI}$ : 0.49-0.73; cetuximab vs. endostar: $\mathrm{HR}=2.25,95 \% \mathrm{CrI}$ : 1.83-2.77; cetuximab vs. lapatinib: $\mathrm{HR}=1.33,95 \% \mathrm{CrI}$ : 1.11-1.60; cetuximab vs. ramucirumab: $\mathrm{HR}=2.24,95 \%$ CrI: 1.69-2.96; cetuximab vs. trastuzumab: $\mathrm{HR}=1.53$, 95\% CrI: 1.23-1.89). 


\section{Adverse effect}

With respect to fatigue, onartuzumab was reported to be associated with relatively low incidence rate compared to lapatinib ( $\mathrm{OR}=0.07,95 \% \mathrm{CrI}$ : $0.00-0.89$ ), and ramucirumab was the only one performing worse than placebo $(\mathrm{OR}=1.90,95 \% \mathrm{CrI}: 1.02-2.94)$.

In terms of neutropenia, patients treated with everolimus and lapatinib reported higher incidence rate than those treated with bevacizumab $(\mathrm{OR}=13.60,95 \% \mathrm{CrI}$ : 1.80-399.41; OR $=3.90,95 \%$ CrI: $1.57-11.82)$, cetuximab $(\mathrm{OR}=16.61,95 \% \mathrm{CrI}: 2.20-497.70 ; \mathrm{OR}=4.85,95 \% \mathrm{CrI}$ : 1.92-13.60), endostar ( $\mathrm{OR}=12.55,95 \% \mathrm{CrI}: 1.42-407.48$; $\mathrm{OR}=3.63,95 \% \mathrm{CrI}: 14.15)$, trastuzumab $(\mathrm{OR}=12.30$, 95\%CrI: $1.60-368.71$; OR $=3.63,95 \%$ CrI: $1.34-10.18$ ), and even placebo (OR $=12.30,95 \% \mathrm{CrI}$ : $1.82-350.72$; OR $=3.60,95 \%$ CrI: $1.77-8.50$, respectively). Ramucirumab yielded similar results, with higher risk of neutropenia compared with placebo (OR $=2.89,95 \% \mathrm{CrI}: 2.08-4.22)$, bevacizumab (OR $=3.10,95 \% \mathrm{CrI}$ : $1.60-6.75)$, cetuximab $(\mathrm{OR}=3.90,95 \% \mathrm{CrI}: 1.92-7.85)$ and trastuzumab $(\mathrm{OR}=2.92,95 \% \mathrm{CrI}: 1.31-5.93)$.

As for diarrhoea, some treatments performed worse than placebo, including lapatinib $(\mathrm{OR}=5.70,95 \% \mathrm{CrI}$ : 2.48-14.73), ramucirumab (OR $=2.39,95 \%$ CrI: 1.22 4.76), trastuzumab ( $\mathrm{OR}=2.64,95 \% \mathrm{CrI}: 1.02-7.24)$, while onartuzumab showed significant superiority to lapatinib $(\mathrm{OR}=0.24,95 \%$ CrI: 0.06-0.92), which was consistent with the result of fatigue.
In the meanwhile, no statistical difference was found in terms of anaemia, vomiting and nausea.

\section{Ranking analysis}

To help us with a better understanding of the results, we plotted the cumulative ranking probability curves to calculate SUCRA value for all medications on investigated outcomes. As illustrated in Table 1, ramucirumab showed a high efficacy in all survival outcomes except for the lack of 3y-OS as well as trastuzumab was efficacious in all OS and 1y-PFS. Lapatinib was another good treatment for improving OS, while endostar and everolimus were effective agents for PFS items. For secondary outcomes, Disappointingly, almost all treatments ranked lower than placebo. However, exceptions existed. Cetuximab was associated with lower risk of neutropenia and nausea than all other treatments, ramucirumab exhibited better performance in terms of anaemia and vomiting compared to other treatments, while onartuzumab and bevacizumab were the effective treatment in controlling fatigue and diarrhoea respectively.

\section{Jadad scale}

The Jadad Scale of included studies demonstrated in Supplementary Table 4 showed that all trails were randomized and controlled, but unfortunately, the majority of included studies were open-label trials and only a few adopted double-

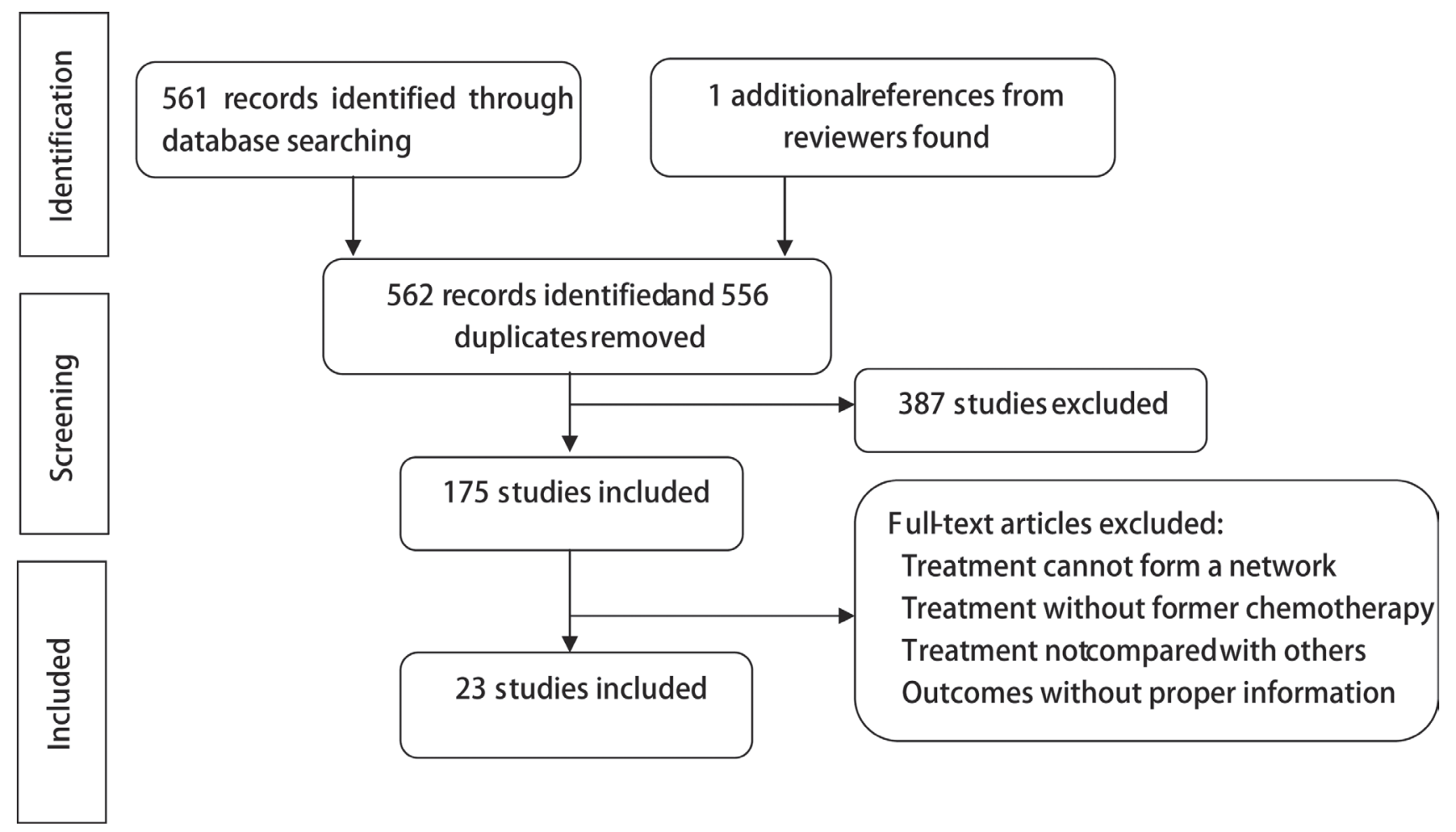

Figure 1: Flow chart of included studies. 
blinding methods, which might add to the heterogeneity of this NMA. Also, as was expected, there were withdrawals in all studies, partly due to the intensity of chemotherapy.

\section{DISCUSSION}

In the current analysis, we assessed the efficacy and safety of common targeted agents used in combination with chemotherapy. including 12 regimens on 7 targets, i.e. HER2 (trastuzumab, lapatinib), EGFR (nimotuzumab, panitumumab, matuzumab, cetuximab), VEGF (bevacizumab, endostar), VEGFR (ramucirumab), TKI (sunitinib), HGFR(onartuzumab) and mTOR (everolimus).

Human epidermal growth factor receptor-2 (HER2) mutation is one prevailing mutation in the pathogenesis of gastric cancer [32]. Trastuzumab is a monoclonal anti-HER2 antibody, whereas lapatinib is a tyrosine kinase inhibitor of both ERB1 and HER2. For HER2 mutation, we found that both trastuzumab and lapatinib were effective in OS and trastuzumab was also good in short-term PFS, yet lapatinib had a higher risk of adverse events and trastuzumab exhibited mediocre performace in controlling all adverse events except neutropenia. Actually, trastuzumab has been reported to be effective in combination with first-line fluoropyrimidine and cisplatin therapy [33]. And the addition of trastuzumab to chemotherapy for gastric and gastroesophageal cancer significantly yielded survival benefits compared to other antibodies [34]. It was also reported that the association of trastuzumab with oxaliplatin and fluoropyrimidin could reduce the toxicity of therapy compared with the addition of fluoropyrimidin and cisplatin [35], which was consistent with the results of our NMA to some extent.

Vascular endothelial growth factor (VEGF) and its receptors (VEGFR) play critical roles in the angiogenesis and metastasis of gastric cancer [36]. Ramucirumab is a monoclonal anti-VEGFR-2 antibody and bevacizumab is

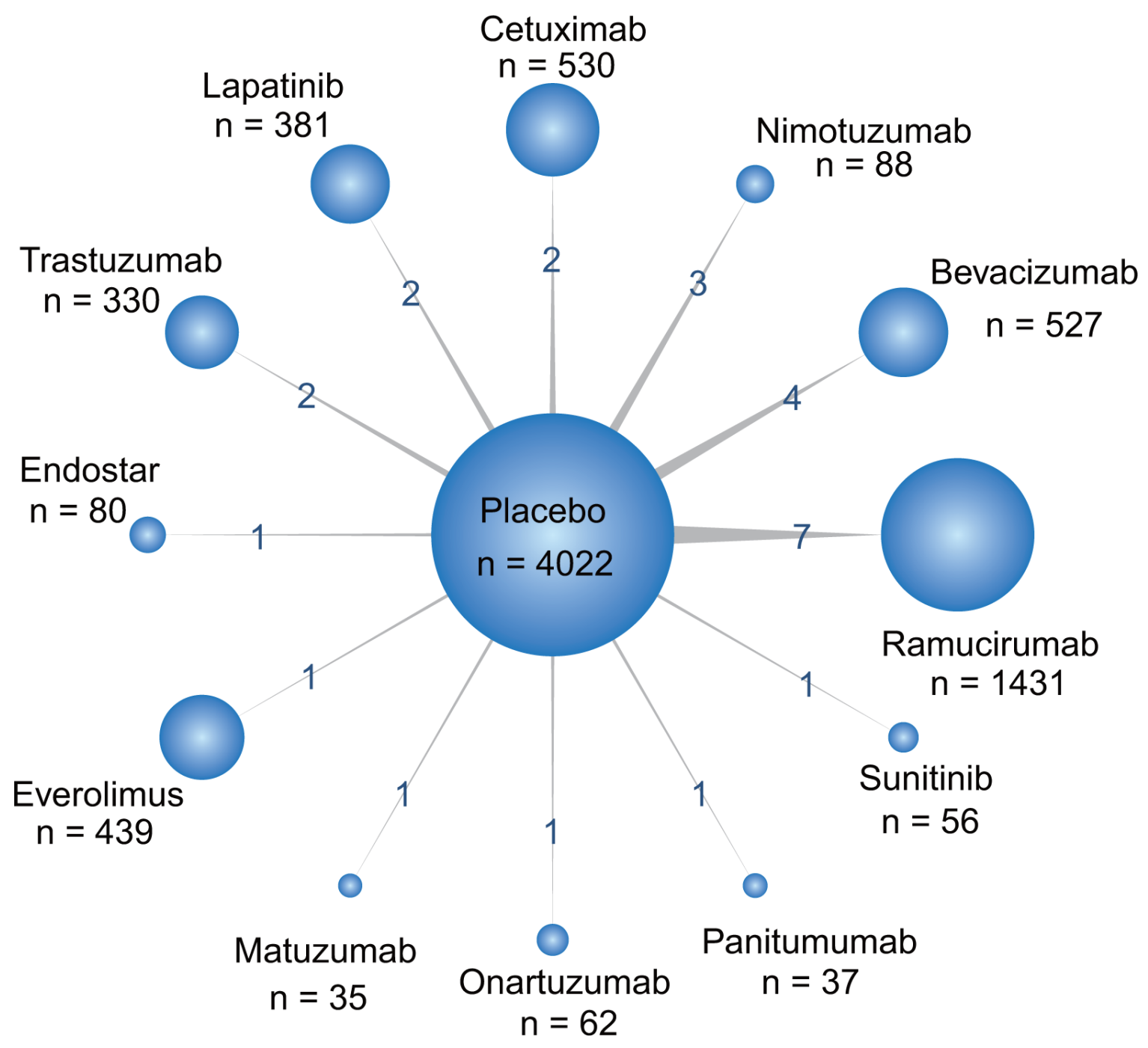

Figure 2: Network of all randomized controlled trials comparing primary outcomes of different targeted therapies for gastric cancer. The width of the lines is proportional to the number of trials comparing each pair of treatments with numbers on the lines illustrating the exact number; the area of circles represents the cumulative number of patients for each intervention. 
the antibody of VEGF-A, both widely used in the treatment of lung, breast, and renal cancer. According to our study, ramucirumab performed extraordinarily well in all survival terms, which was consistent with the results of previously reported studies [37], and was associated with relative low risk of anaemia, neutropenia and vomiting, though the results with regard to fatigue, neutropenia and diarrhoea were not desirable. As for bevacizumab, although was not as effective as ramucirumab, still yielded relatively good results in improving survival rate while caused few adverse events according to this NMA. Yet according to previous RCTs, bevacizumab showed no significant benefit on improving survival rate $[26,38]$, so its performance need to be further evaluated in future studies.

\section{1y-Overall Survival}

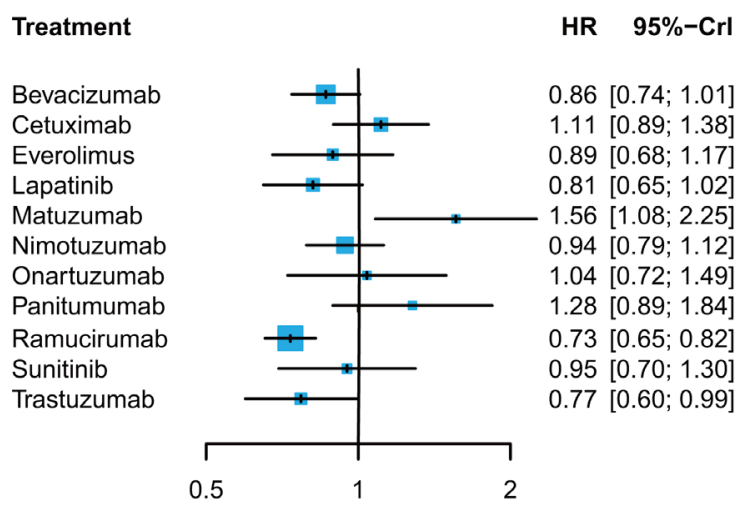

2y-Overall Survival

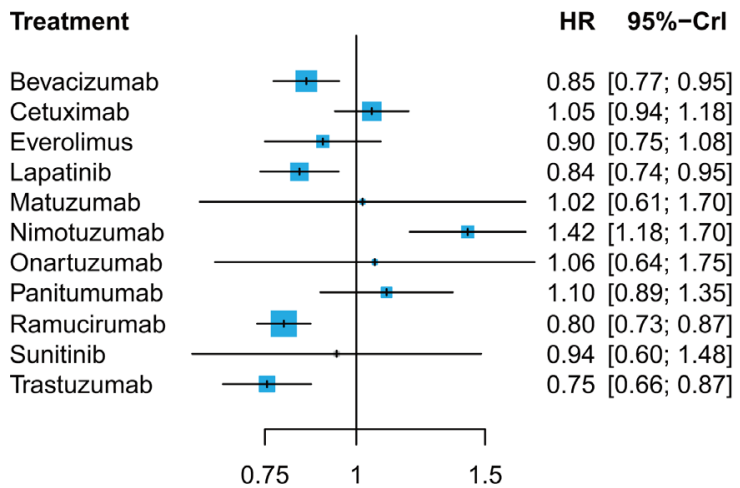

3y- Overall Survival

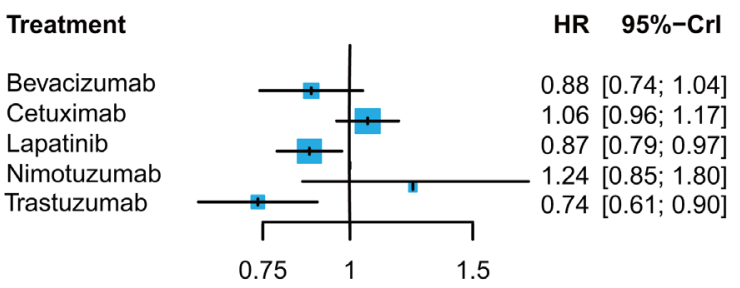

Mutations of epidermal growth factor receptor (EGFR) contribute to the growth and metastasis of tumor. Nimotuzumab, panitumumab, matuzumab and cetuximab are all antibodies of EGFR. By inhibiting the over-expression of EGFR, they help to restrict the growth, invasion and metastasis of tumor. According to our results, the efficacy of anti-EGFR treatments was not superior to other therapies. Nimotuzumab and matuzumab were even less efficacious than placebo in some outcomes. Also there was one clinical report suggested that the addition of nimotuzumab weakened the antitumor effect of cisplatin regimen, which is the first line chemotherapy for patients, and this lead to a worse efficacy than the addition of placebo [24]. Although EGFR may not be

\section{1y-Progession-Free Survival}

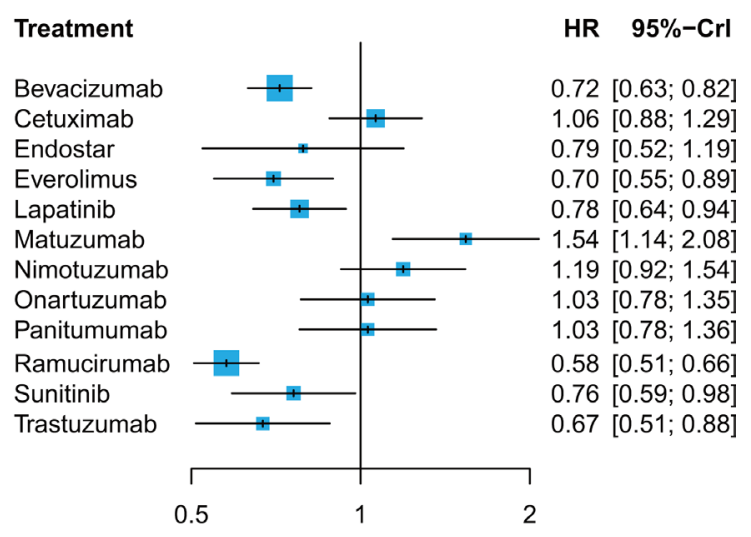

2y-Progession-Free Survival

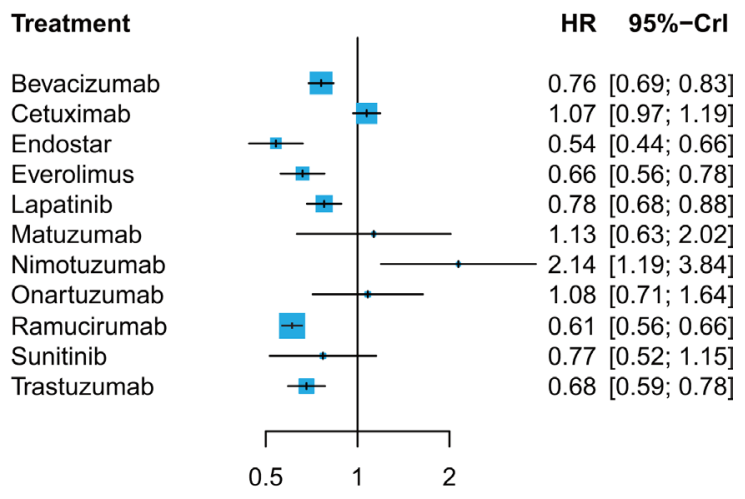

\section{3y-Progession-Free Survival}

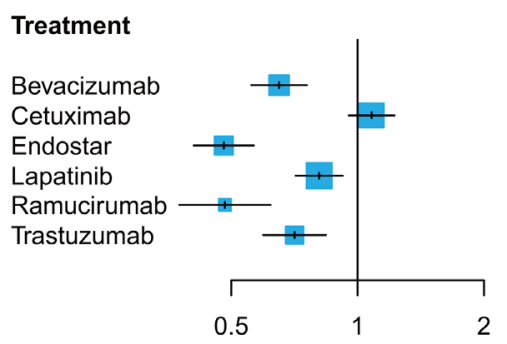

HR $95 \%-\mathrm{Crl}$

$0.65[0.56 ; 0.76]$

$1.08[0.95 ; 1.22]$

$0.48[0.41 ; 0.57]$

$0.81[0.71 ; 0.92]$

0.48 [0.38; 0.62$]$

$0.71[0.60 ; 0.84]$

Figure 3: Hazard ratios (95\% credible intervals) for network comparison of primary outcomes for gastric cancer treatments. 
beneficial for the survival of patients with gastric cancer, they were found to have significant predictive ability for the prognosis of patients [39].

Tyrosine kinase (TK) works downstream of EGFR and VEGFR in the same pathway [40]. Sunitinib inhibits cellular signaling by targeting multiple receptor tyrosine kinases including platelet-derived growth factor receptors (PDGF-Rs) and VEGFRs. However, according to this NMA, although not the worst, the performance of sunitinib was nor outstanding in either improving survival rate or controlling adverse events.

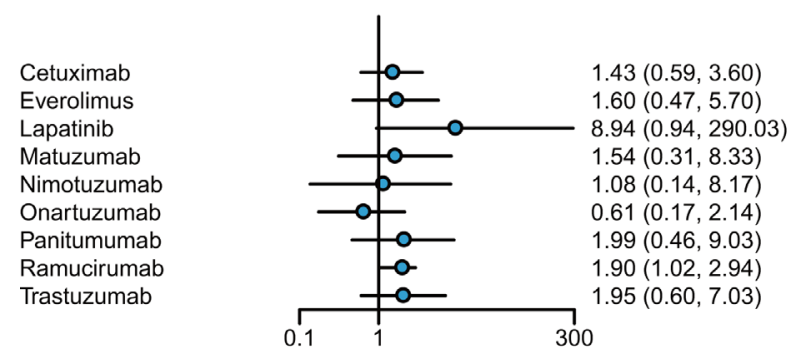

Fatigue

Compared with Placebo

Bevacizumab
Cetuximab
Endostar
Everolimus
Lapatinib
Matuzumab
Nimotuzumab
Onartuzumab
Panitumumab
Ramucirumab
Sunitinib
Trastuzumab

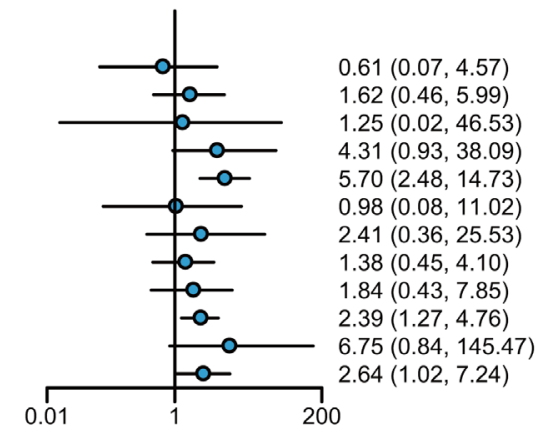

Diarrhoea
Bevacizumab

Cetuximab

Endostar

Everolimus

Lapatinib

Matuzumab

Nimotuzumab

Ramucirumab

Sunitinib

Trastuzumab

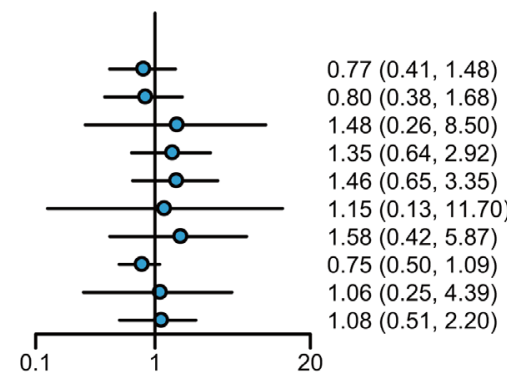

Anaemia

Compared with Placebo

Odds Ratio (95\% Crl)

Bevacizumab

Cetuximab

Everolimus

Lapatinib

Matuzumab

Nimotuzumab

Onartuzumab

Panitumumab

Ramucirumab

Sunitinib

Trastuzumab

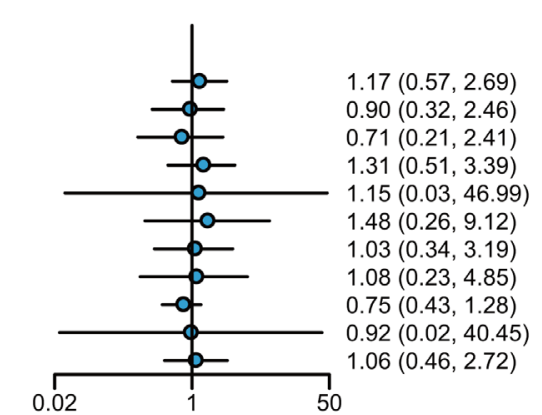

Vomiting

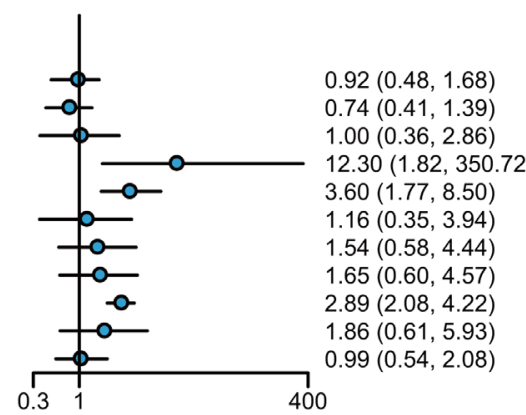

Neutropenia
Bevacizumab

Cetuximab

Endostar

Everolimus

Lapatinib

Matuzumab

Nimotuzumab

Onartuzumab

Panitumumab

Ramucirumab

Sunitinib

Trastuzumab
Odds Ratio (95\% Crl)

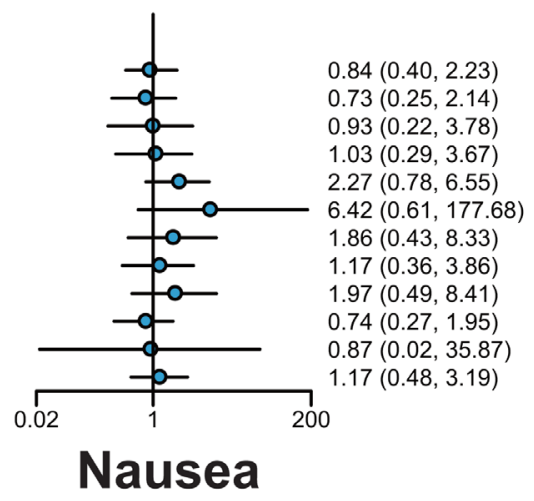

Figure 4: Odds ratios (95\% credible intervals) for network comparison of secondary outcomes for gastric cancer treatments. 
Table 1: SUCRA value of treatments for gastric cancer therapy

\begin{tabular}{|c|c|c|c|c|c|c|c|c|c|c|c|c|}
\hline & 1-OS & 2-OS & 3-OS & 1-PFS & 2-PFS & 3-PFS & Fatigue & Anaemia & Neutropenia & Diarrhoea & Vomiting & Nausea \\
\hline Placebo & 0.384 & 0.387 & 0.364 & 0.331 & 0.300 & 0.148 & 0.747 & 0.523 & 0.694 & 0.785 & 0.502 & 0.588 \\
\hline Bevacizumab & 0.673 & 0.681 & 0.683 & 0.735 & 0.561 & 0.630 & - & 0.726 & 0.746 & 0.819 & 0.400 & 0.674 \\
\hline Cetuximab & 0.252 & 0.294 & 0.192 & 0.249 & 0.203 & 0.019 & 0.531 & 0.695 & 0.870 & 0.578 & 0.570 & 0.743 \\
\hline Endostar & - & - & - & 0.609 & 0.972 & 0.919 & - & 0.377 & 0.686 & 0.608 & - & 0.611 \\
\hline Everolimus & 0.607 & 0.585 & - & 0.761 & 0.767 & - & 0.474 & 0.331 & 0.036 & 0.253 & 0.675 & 0.570 \\
\hline Lapatinib & 0.760 & 0.727 & 0.708 & 0.627 & 0.532 & 0.354 & 0.089 & 0.302 & 0.142 & 0.147 & 0.353 & 0.237 \\
\hline Matuzumab & 0.036 & 0.415 & - & 0.015 & 0.238 & - & 0.501 & 0.478 & 0.611 & 0.691 & 0.475 & 0.115 \\
\hline Nimotuzumab & 0.511 & 0.032 & 0.087 & 0.146 & 0.010 & - & 0.625 & 0.320 & 0.471 & 0.440 & 0.359 & 0.338 \\
\hline Onartuzumab & 0.373 & 0.366 & - & 0.295 & 0.241 & - & 0.876 & - & 0.445 & 0.643 & 0.488 & 0.507 \\
\hline Panitumumab & 0.148 & 0.248 & - & 0.295 & - & - & 0.392 & - & - & 0.531 & 0.476 & 0.306 \\
\hline Ramucirumab & 0.923 & 0.835 & - & 0.969 & 0.881 & 0.909 & 0.377 & 0.769 & 0.202 & 0.424 & 0.706 & 0.734 \\
\hline Sunitinib & 0.501 & 0.519 & - & 0.660 & 0.571 & - & - & 0.503 & 0.402 & 0.189 & 0.525 & 0.581 \\
\hline Trastuzumab & 0.832 & 0.912 & 0.966 & 0.808 & 0.726 & 0.522 & 0.389 & 0.477 & 0.695 & 0.392 & 0.471 & 0.497 \\
\hline
\end{tabular}

everolimus, an mTOR inhibitor, was found to be efficacious in 1y-PFS and 2y-PFS. However, the result was denied by previous studies. Therefore, further clinical data was needed for the verification of our result.

Some limitation should be addressed before the application of our results. Firstly, the results can easily be diluted by the lack of evidence. For example, most of the treatments, including endostar, everolimus, matuzumab, onartuzumab, panitumumab and sunitinib were respectively covered by just one study and the number of patients involving in some treatments was relatively small Secondly, in addition to the variety of agents, the regimens of chemotherapy could also affect the prognosis of patients while In this NMA, we did not discriminate the chemotherapy that targeted drugs were combined with, which may add heterogeneity to this study. Moreover, alongside with gastric cancer, some patients with gastro-esophageal cancer were also included in our study, while may also cast doubt on the reliability of this NMA. Nonetheless, despite all these, this is the very first NMA comparing the efficacy and efficacy of different agents, and the selection criteria guaranteed the reliability of included studies.

In the current analysis, we evaluated the efficacy and adverse events of targets agents for gastric cancer. Ramucirumab was efficacious during one year to three years survival rate. However, high risk of hematological events should be noted during the application. Lapatinib was another extraordinary efficacious treatment in improving the overall survival condition, yet the severe adverse events made it not very recommended. Trastuzumab showed high efficacy in improving survival rate and was associated with relatively mild adverse events, therefore, it was recommended as the optimal targeted agent in clinical application. Meanwhile, physicians should consider the mutation of patients, the efficacy of agents, response rate, and side effects to select appropriate treatment.

\section{MATERIALS AND METHODS}

\section{Study selection}

We conducted our literature in PubMed, Embase, the Cochrane Library and Scopus. Literatures published between January 1st 2000 and October 1st 2016 were enrolled in our analysis. To start with, relevant studies were identified using key words "gastric cancer", "chemotherapy", "ramucirumab", "bevacizumab", "nimotuzumab", "cetuximab", "lapatinib", "trastuzumab", "endostar", "everolimus", "matuzumab", "onartuzumab", "panitumumab" and "sunitinib". Irrelevant and duplicate articles were subsequently excluded by screening titles and abstracts. Two authors also reviewed the reference lists of enrolled articles for related study.

We analyzed the full text of retrieved articles. Studies in accordance with our inclusion criteria were enrolled for further analysis. Inclusion criteria: (1) study has to be a randomized clinical trial (RCT); (2) pathological diagnosis of gastric cancer should be confirmed in the study; (3) subjects should be aged from 18 to 80 ; (4) patients should be diagnosed with untreated advanced gastric cancer(5) target agents should be applied in combination with chemotherapy. Exclusion criteria: (1) treatment cannot form a network; (2) treatment lacked former chemotherapy; (3) treatment does not compare with others; (4) outcomes lacked sufficient information. 
Moreover, all included articles were evaluated by Jadad scale [42]. Methods of studies were graded by randomization, blind method and withdrawal. Only studies with relatively high Jadad score were enrolled for further analysis.

\section{Data extraction}

Two authors independently extracted related information from enrolled articles, including last name of first author, year of publication, country, followup duration (in month), sample size, average age of all subjects, targeted agents and their targets. Primary outcomes include 1 year overall survival (1y-OS), 2 year overall survival (2y-OS), 3 year overall survival (3y-OS), 1 year progression-free survival (1y-PFS), 2 year progression-free survival $(2 y-P F S)$ and 3 year progression-free survival (3y-PFS). OS was defined as the time from random diagnosis of gastric cancer to death of patients or the latest date known to be alive for censored patients. Progression-free survival (PFS) was defined as the time from diagnosis to first documented progression. Secondary outcomes are adverse events, including fatigue, anaemia, vomiting, diarrhoea, nausea and neutropenia. Discrepancies between two authors were resolved by a third author after discussion.

\section{Data analysis}

In the current study, we performed a network metaanalysis to assess the outcomes of commonly used targeted agents in treating gastric cancer.

Our network meta-analysis is performed with a Bayesian model in WinBUGS (MRC Bio-statistics Unit, Cambridge, UK). Primary outcomes including 1y-OS, $2 y-$ OS, 3y-OS, 1y-PFS, 2y-PFS and 3y-PFS were represented by hazard ratio (HR) with $95 \%$ corresponding credible interval (CrI); adverse events were represented by odds ratio (OR) with 95\% corresponding CrI. Moreover, we calculated the surface under the cumulative ranking curve (SUCRA) to illustrate the rank probability of each agent for different outcomes based on the result of network meta-analysis.

\section{CONFLICTS OF INTEREST}

The authors declare no conflicts of interest.

\section{FUNDING}

Research on chemotherapy drug resistance mechanism of stem cells in gastric carcinoma mediated by EZH2/FBXO32, 162300410101.

\section{REFERENCES}

1. Torre LA, Bray F, Siegel RL, Ferlay J, Lortet-Tieulent J, Jemal A. Global cancer statistics, 2012. CA Cancer J Clin. 2015; 65:87-108.
2. Soerjomataram I, Lortet-Tieulent J, Parkin DM, Ferlay J, Mathers C, Forman D, Bray F. Global burden of cancer in 2008: a systematic analysis of disability-adjusted life-years in 12 world regions. Lancet. 2012; 380:1840-1850.

3. De Angelis R, Sant M, Coleman MP, Francisci S, Baili P, Pierannunzio D, Trama A, Visser O, Brenner H, Ardanaz E, Bielska-Lasota M, Engholm G, Nennecke A, et al. Cancer survival in Europe 1999-2007 by country and age: results of EUROCARE-5-a population-based study. Lancet Oncol. 2014; 15:23-34.

4. Fuchs CS, Tomasek J, Yong CJ, Dumitru F, Passalacqua R, Goswami C, Safran H, dos Santos LV, Aprile G, Ferry DR, Melichar B, Tehfe M, Topuzov E, et al. Ramucirumab monotherapy for previously treated advanced gastric or gastro-oesophageal junction adenocarcinoma (REGARD): an international, randomised, multicentre, placebocontrolled, phase 3 trial. Lancet. 2014; 383:31-39.

5. Ford HE, Marshall A, Bridgewater JA, Janowitz T, Coxon FY, Wadsley J, Mansoor W, Fyfe D, Madhusudan S, Middleton GW, Swinson D, Falk S, Chau I, et al. Docetaxel versus active symptom control for refractory oesophagogastric adenocarcinoma (COUGAR-02): an open-label, phase 3 randomised controlled trial. Lancet Oncol. 2014; 1:78-86.

6. de Mestier L, Lardiere-Deguelte S, Volet J, Kianmanesh R, Bouche O. Recent insights in the therapeutic management of patients with gastric cancer. Dig Liver Dis. 2016; 48:984-994.

7. Kang JH, Lee SI, Lim DH, Park KW, Oh SY, Kwon HC, Hwang IG, Lee SC, Nam E, Shin DB, Lee J, Park JO, Park YS, et al. Salvage chemotherapy for pretreated gastric cancer: a randomized phase III trial comparing chemotherapy plus best supportive care with best supportive care alone. J Clin Oncol. 2012; 30:1513-1518.

8. Moja L, Tagliabue L, Balduzzi S, Parmelli E, Pistotti V, Guarneri V, D’Amico R. Trastuzumab containing regimens for early breast cancer. Cochrane Database Syst Rev. 2012; CD006243.

9. Bang YJ, Van Cutsem E, Feyereislova A, Chung HC, Shen L, Sawaki A, Lordick F, Ohtsu A, Omuro Y, Satoh T, Aprile G, Kulikov E, Hill J, et al. Trastuzumab in combination with chemotherapy versus chemotherapy alone for treatment of HER2-positive advanced gastric or gastrooesophageal junction cancer (ToGA): A phase 3, open-label, randomised controlled trial. Lancet. 2010; 376:687-697.

10. Rao S, Starling N, Cunningham D, Sumpter K, Gilligan D, Ruhstaller T, Valladaresayerbes M, Wilke H, Archer C, Kurek R. Matuzumab plus epirubicin, cisplatin and capecitabine (ECX) compared with epirubicin, cisplatin and capecitabine alone as first-line treatment in patients with advanced oesophago-gastric cancer: a randomised, multicentre open-label phase II study. Ann Oncol. 2010; 21:2213-2219.

11. Ohtsu A, Shah MA, Van Cutsem E, Rha SY, Sawaki A, Park SR, Lim HY, Yamada Y, Wu J, Langer B, 
Starnawski M, Kang YK. Bevacizumab in combination with chemotherapy as first-line therapy in advanced gastric cancer: A randomized, double-blind, placebo-controlled phase III study. J Clin Oncol. 2011; 29:3968-3976.

12. Van Cutsem E, De Haas S, Kang YK, Ohtsu A, Tebbutt NC, $\mathrm{Xu}$ JM, Yong WP, Langer B, Delmar P, Scherer SJ, Shah MA. Bevacizumab in combination with chemotherapy as first-line therapy in advanced gastric cancer: A biomarker evaluation from the AVAGAST randomized phase III trial. J Clin Oncol. 2012; 30:2119-2127.

13. Yi JH, Lee J, Lee J, Park SH, Park JO, Yim DS, Park YS, Lim HY, Kang WK. Randomised phase II trial of docetaxel and sunitinib in patients with metastatic gastric cancer who were previously treated with fluoropyrimidine and platinum. Br J Cancer. 2012; 106:1469-1474.

14. Lordick F, Kang YK, Chung HC, Salman P, Oh SC, Bodoky G, Kurteva G, Volovat C, Moiseyenko VM, Gorbunova V, Park JO, Sawaki A, Celik I, et al. Capecitabine and cisplatin with or without cetuximab for patients with previously untreated advanced gastric cancer (EXPAND): a randomised, open-label phase 3 trial. Lancet Oncol. 2013; 14:490-499.

15. Ohtsu A, Ajani JA, Bai YX, Bang YJ, Chung HC, Pan HM, Sahmoud T, Shen L, Yeh KH, Chin K, Muro K, Kim YH, Ferry D, et al. Everolimus for previously treated advanced gastric cancer: results of the randomized, double-blind, phase III GRANITE-1 study. J Clin Oncol. 2013; 31:3935-3943.

16. Richards D, Kocs DM, Spira AI, David McCollum A, Diab S, Hecker LI, Cohn A, Zhan F, Asmar L. Results of docetaxel plus oxaliplatin (DOCOX) $+/-$ cetuximab in patients with metastatic gastric and/or gastroesophageal junction adenocarcinoma: results of a randomised Phase 2 study. Eur J Cancer. 2013; 49:2823-2831.

17. Shen L, Xu J, Feng F, Jiao S, Wang L, Li J, Guan ZZ, Qin S, Wang J, Yu S, Wang Y, Jin Y, Tao M, et al. [Trastuzumab in combination with chemotherapy versus chemotherapy alone for first-line treatment of HER2-positive advanced gastric or gastroesophageal junction cancer: a Phase III, multi-center, randomized controlled trial, Chinese subreport]. [Article in Chinese]. Zhonghua zhong liu za zhi. 2013; 35:295-300.

18. Xu R, Ma N, Wang F, Ma L, Chen R, Chen R, Kebinu M, Ma L, Han Z, Ayixiamu, Mayier M, Su P, Naman Y, et al. Results of a randomized and controlled clinical trial evaluating the efficacy and safety of combination therapy with Endostar and S-1 combined with oxaliplatin in advanced gastric cancer. OncoTargets Ther. 2013; 6:925-929.

19. Fuchs CS, Tomasek J, Yong CJ, Dumitru F, Passalacqua R, Goswami C, Safran H, dos Santos LV, Aprile G, Ferry DR, Melichar B, Tehfe M, Topuzov E, et al. Ramucirumab monotherapy for previously treated advanced gastric or gastro-oesophageal junction adenocarcinoma (REGARD): an international, randomised, multicentre, placebocontrolled, phase 3 trial. Lancet. 2014; 383:31-39.

20. Satoh T, Xu RH, Chung HC, Sun GP, Doi T, Xu JM, Tsuji A, Omuro Y, Li J, Wang JW, Miwa H, Qin SK, Chung IJ, et al.
Lapatinib plus paclitaxel versus paclitaxel alone in the second-line treatment of HER2-amplified advanced gastric cancer in Asian populations: TyTAN_ - a randomized, phase III study. J Clin Oncol. 2014; 32:2039-2049.

21. Wilke H, Muro K, Van Cutsem E, Oh SC, Bodoky G, Shimada Y, Hironaka S, Sugimoto N, Lipatov O, Kim TY, Cunningham D, Rougier P, Komatsu Y, et al. Ramucirumab plus paclitaxel versus placebo plus paclitaxel in patients with previously treated advanced gastric or gastrooesophageal junction adenocarcinoma (RAINBOW): a double-blind, randomised phase 3 trial. Lancet Oncol. 2014; 15:1224-1235.

22. Xu CD. Clinical study of nimotuzumab combined with chemotherapy in the treatment of late stage gastric cancer. Asian Pac J Cancer Prev. 2014; 15:10273-10276.

23. Casak SJ, Fashoyin-Aje I, Lemery SJ, Zhang L, Jin R, Li H, Zhao L, Zhao H, Zhang H, Chen H, He K, Dougherty M, Novak R, et al. FDA Approval Summary: Ramucirumab for Gastric Cancer. Clin Cancer Res. 2015; 21:3372-3376.

24. Du F, Zheng Z, Shi S, Jiang Z, Qu T, Yuan X, Sun Y, Song Y, Yang L, Zhao J, Wang J, Chi Y. S-1 and Cisplatin With or Without Nimotuzumab for Patients With Untreated Unresectable or Metastatic Gastric Cancer: A Randomized, Open-Label Phase 2 Trial. Medicine. 2015; 94:e958.

25. Satoh T, Lee KH, Rha SY, Sasaki Y, Park SH, Komatsu Y, Yasui H, Kim TY, Yamaguchi K, Fuse N, Yamada Y, Ura T, Kim SY, et al. Randomized phase II trial of nimotuzumab plus irinotecan versus irinotecan alone as second-line therapy for patients with advanced gastric cancer. Gastric Cancer. 2015; 18:824-832.

26. Shen L, Li J, Xu J, Pan H, Dai G, Qin S, Wang L, Wang J, Yang Z, Shu Y, Xu R, Chen L, Liu Y, et al. Bevacizumab plus capecitabine and cisplatin in Chinese patients with inoperable locally advanced or metastatic gastric or gastroesophageal junction cancer: randomized, double-blind, phase III study (AVATAR study). Gastric Cancer. 2015; 18:168-176.

27. Hecht JR, Bang YJ, Qin SK, Chung HC, Xu JM, Park JO, Jeziorski K, Shparyk Y, Hoff PM, Sobrero A, Salman P, Li J, Protsenko SA, et al. Lapatinib in combination with capecitabine plus oxaliplatin in human epidermal growth factor receptor 2-positive advanced or metastatic gastric, esophageal, or gastroesophageal adenocarcinoma: TRIO013/LOGiC - A randomized phase III trial. J Clin Oncol. 2016; 34:443-451.

28. Muro K, Oh SC, Shimada Y, Lee KW, Yen CJ, Chao Y, Cho JY, Cheng R, Carlesi R, Chandrawansa K, Orlando M, Ohtsu A. Subgroup analysis of East Asians in RAINBOW: A phase 3 trial of ramucirumab plus paclitaxel for advanced gastric cancer. J Gastroenterol Hepatol. 2016; 31:581-589.

29. Shah MA, Cho JY, Tan IB, Tebbutt NC, Yen CJ, Kang A, Shames DS, Bu L, Kang YK. A Randomized Phase II Study of FOLFOX With or Without the MET Inhibitor Onartuzumab in Advanced Adenocarcinoma of the Stomach and Gastroesophageal Junction. Oncologist. 2016; 21:1085-90. 
30. Shitara K, Muro K, Shimada Y, Hironaka S, Sugimoto N, Komatsu Y, Nishina T, Yamaguchi K, Segawa Y, Omuro Y, Tamura T, Doi T, Yukisawa S, et al. Subgroup analyses of the safety and efficacy of ramucirumab in Japanese and Western patients in RAINBOW: a randomized clinical trial in second-line treatment of gastric cancer. Gastric Cancer. 2016; 19:927-938.

31. Tebbutt NC, Price TJ, Ferraro DA, Wong N, Veillard AS, Hall M, Sjoquist KM, Pavlakis N, Strickland A, Varma SC, Cooray P, Young R, Underhill C, et al. Panitumumab added to docetaxel, cisplatin and fluoropyrimidine in oesophagogastric cancer: ATTAX3 phase II trial. Br J Cancer. 2016; 114:505-509.

32. Peng Z, Zou J, Zhang X, Yang Y, Gao J, Li Y, Li Y, Shen L. HER2 discordance between paired primary gastric cancer and metastasis: a meta-analysis. Chinese J Cancer Res. 2015; 27:163-171.

33. Bang YJ, Van Cutsem E, Feyereislova A, Chung HC, Shen L, Sawaki A, Lordick F, Ohtsu A, Omuro Y, Satoh T, Aprile G, Kulikov E, Hill J, et al. Trastuzumab in combination with chemotherapy versus chemotherapy alone for treatment of HER2-positive advanced gastric or gastrooesophageal junction cancer (ToGA): a phase 3, open-label, randomised controlled trial. Lancet. 2010; 376:687-697.

34. Luo HQ, Han L, Jiang Y. Meta-analysis of six randomized control trials of chemotherapy plus anti-HER monoclonal antibody for advanced gastric and gastroesophageal cancer. Asian Pac J Cancer Prev. 2014; 15:5343-5348.

35. Ryu MH, Yoo C, Kim JG, Ryoo BY, Park YS, Park SR, Han HS, Chung IJ, Song EK, Lee KH, Kang SY, Kang YK. Multicenter phase II study of trastuzumab in combination with capecitabine and oxaliplatin for advanced gastric cancer. Eur J Cancer. 2015; 51:482-488.

36. Oh SY, Kwon HC, Kim SH, Jang JS, Kim MC, Kim KH, Han JY, Kim CO, Kim SJ, Jeong JS, Kim HJ. Clinicopathologic significance of HIF-1alpha, p53, and VEGF expression and preoperative serum VEGF level in gastric cancer. BMC cancer. 2008; 8:123.
37. Wilke H, Muro K, Van Cutsem E, Oh SC, Bodoky G, Shimada Y, Hironaka S, Sugimoto N, Lipatov O, Kim TY, Cunningham D, Rougier P, Komatsu Y, et al. Ramucirumab plus paclitaxel versus placebo plus paclitaxel in patients with previously treated advanced gastric or gastrooesophageal junction adenocarcinoma (RAINBOW): a double-blind, randomised phase 3 trial. Lancet Oncol. 2014; 15:1224-1235.

38. Ohtsu A, Shah MA, Van Cutsem E, Rha SY, Sawaki A, Park SR, Lim HY, Yamada Y, Wu J, Langer B, Starnawski M, Kang YK. Bevacizumab in combination with chemotherapy as first-line therapy in advanced gastric cancer: a randomized, double-blind, placebo-controlled phase III study. J Clin Oncol. 2011; 29:3968-3976.

39. Chen C, Yang JM, Hu TT, Xu TJ, Yan G, Hu SL, Wei W, $\mathrm{Xu}$ WP. Prognostic role of human epidermal growth factor receptor in gastric cancer: a systematic review and metaanalysis. Arch Med Res. 2013; 44:380-389.

40. Habib AA, Chatterjee S, Park SK, Ratan RR, Lefebvre S, Vartanian T. The epidermal growth factor receptor engages receptor interacting protein and nuclear factor-kappa B (NF-kappa B)-inducing kinase to activate NF-kappa B. Identification of a novel receptor-tyrosine kinase signalosome. J Biol Chem. 2001; 276:8865-8874.

41. Shah MA, Cho JY, Tan IB, Tebbutt NC, Yen CJ, Kang A, Shames DS, Bu L, Kang YK. A Randomized Phase II Study of FOLFOX With or Without the MET Inhibitor Onartuzumab in Advanced Adenocarcinoma of the Stomach and Gastroesophageal Junction. Oncologist. 2016; 21:1085-1090.

42. Jadad AR, Moore RA, Carroll D, Jenkinson C, Reynolds DJ, Gavaghan DJ, McQuay HJ. Assessing the quality of reports of randomized clinical trials: is blinding necessary? Control Clin Trials. 1996; 17:1-12. 